Research Article

\title{
Formulation, Characterization, in vitro Anti-Tubercular Activity and Cytotoxicity Study of Solid Lipid Nano- particles of Isoniazid
} \author{
Vijayan Nynar Azger Dusthackeer ${ }^{6}$ \\ ${ }^{1}$ School of Pharmaceutical Education and Research, Berhampur University, Berhampur, Odisha, India. \\ ${ }^{2}$ School of Health Sciences, Mekelle University, Mekelle, Ethiopia. \\ ${ }^{3}$ Faculty of Pharmaceutical Sciences, Rama University, Kanpur, Uttar Pradesh, India. \\ ${ }^{4}$ Department of Zoology, Berhampur University, Berhampur, Odisha, India. \\ ${ }^{5}$ Sree Vidyanikethan College of Pharmacy, Tirupati, Andhra Pradesh, India. \\ ${ }^{6}$ Department of Bacteriology, National Institute of Research in Tuberculosis, Chennai, India. \\ Corresponding author. E-mail: bibhashmohanta@gmail.com
}

Bibhash Chandra Mohanta ${ }^{1}$, Subas Chandra Dinda ${ }^{2,3}$, Gitanjali Mishra ${ }^{4}$, Narahari Narayan Palei ${ }^{5}$,

Received: May 23, 2018; Accepted: Aug. 16, 2018; Published: Nov. 21, 2018.

Citation: Bibhash Chandra Mohanta, Subas Chandra Dinda, Gitanjali Mishra, Narahari Narayan Palei, and Vijayan Nynar Azger Dusthackeer, Formulation, Characterization, in vitro Anti-Tubercular Activity and Cytotoxicity Study of Solid Lipid Nanoparticles of Isoniazid. Nano Biomed. Eng., 2018, 10(4): 379-391.

DOI: $10.5101 /$ nbe.v10i4.p379-391.

\begin{abstract}
The present study was aimed to develop and optimize isoniazid (INZ) loaded solid lipid nanoparticles (SLNs) for exploring in vitro anti-tubercular and cytotoxic activity. The INZ-SLNs were successfully prepared by high pressure homogenization followed by ultrasonication technique and optimized using $2^{3}$ full factorial designs. INZ-SLNs were characterized for particle size (PS), zeta potential (ZP), entrapment efficiency percentage (EE\%) and cumulative percentage drug release (CDR\%). Physicochemical properties were investigated using transmission electron microscopy (TEM), differential scanning calorimeter (DSC), X-ray diffraction and Fourier transmission infrared spectroscopy (FTIR). The average PS, ZP and EE\% of the optimized formulation were found to be $167.1 \mathrm{~nm},-32.4 \mathrm{mV}$ and $73.17 \%$ respectively. The optimized formulation showed a CDR of $79.14 \%$ up to $36 \mathrm{~h}$. In vitro anti-tubercular (luciferase reporter phage (LRP) assay in H37Rv viable and resistant strain) and cytotoxicity efficacy (3-(4,5-dimethylthiazolyl-2)-2,5-diphenyltetrazolium bromide (MTT) assay in J774A.1 cells) of INZ-SLNs were evaluated and compared with free INZ. Results of LRP assay in H37Rv strain showed that percentage reduction in relative light unit (RLU) for INZ-SLNs and free INZ were 99.75 and $99.898 \%$ respectively, whereas in case of INZ resistant strain they were found to be 90.27 and $90.52 \%$ respectively, confirming notable antitubercular activity. MTT assay revealed that the percentage of cell viability upon exposure with INZ-SLNs was significantly higher $(>90 \%)$ than free INZ $(<80 \%)$, confirming its safety. Thus, INZ-SLNs could be an effective dosage form with sustained drug release profile, significant anti-tubercular activity, and reduced normal cell toxicity for achieving better therapeutic activity.
\end{abstract}

Keywords: Isoniazid; SLN; In vitro drug release; Luciferase reporter phase assay; MTT 


\section{Introduction}

Tuberculosis (TB) is a highly infectious pervasive disease of the respiratory system caused by the Mycobacterium tuberculosis (MTb) and represents a substantial health threat to mankind distressing up to $33.33 \%$ of the global population [1]. It was reported by W.H.O. that in 2017, around 1.3 million TB deaths occurred globally in 2016. Additionally 3,70,000 deaths were resulting from co-infection of TB with human immunodeficiency virus (HIV).

In general, current TB therapy needs administration of anti-tubercular drugs (ATDs) for a prolonged period. Moreover, the ATDs with shorter biological half-life $\left(t_{1 / 2}\right)$ needs repeated dosing. Isoniazid (INZ) is the most effective front line ATD that possesses high MTb selectivity and excellent bacteriostatic capacity [2]. But unfortunately, it has shorter biological $\mathrm{t}_{1 / 2}(1-4 \mathrm{~h})$ due to which it needs regular as well as repeated dosing that leads to hepatotoxicity and other unwanted side effects [3]. Occurrence of acquired INZ-resistant TB is also very frequent due to inadequate treatment linked with the extensive hepatic metabolism as well as poorer penetration (due to its highly polar nature) of it through plentiful lipoidal cell wall of MTb [4].

Several attempts were being made to resolve the toxicity, acquired resistant TB, first pass metabolism problems of INZ and to deliver it to the primary site of infection through novel drug delivery system for achieving effective TB therapy. With the rapid development of nanotechnology, introduction of nanoparticles and nanoencapsulation technique in drug delivery systems gain much more interest for the treatment of TB $[5,6]$. Polymeric nanoparticulate systems are reported for INZ to have shown promising results and are believed to overcome most of the limitations of traditional delivery approaches [7]. Regrettably, in long-term therapy, polymeric nanoparticles are not preferred mainly due to carriermediated toxicities which arise from organic solvents consumed during nanoparticle preparation and higher product cost [8].

Solid lipid nanoparticles (SLNs) are proposed as an alternative drug delivery system to polymeric nanoparticles and other colloidal carriers [9], have gained considerable interest since last few decades due to their inherited properties like, biocompatibility and biodegradability, lower toxicity, ability to incorporate both hydrophilic and lipophilic drugs, improved bioavailability, stabilization of drugs [10], controlledrelease characteristics [11], and targeted delivery of drugs. Several researchers have already developed SLNs successfully for treatment of different diseases such as cancer [12], diabetics [13], inflammation [14], Alzheimer's disease [15], etc.

In TB therapy, SLNs also have occupied a special status among all other different drug delivery systems as these help in enhancing the bioavailability of ATDs due to their preferential uptake into the various lymphatic systems of the body through intracellular, intercellular and paracellular uptake and uptake through lipase-arbitrated chylomicron pathway. Furthermore, lipoidal behavior and nano size range of SLNs improve their penetrability of the drug across the lipoidal cell wall of MTb and safeguards it from unwanted metabolism [16].

Several researchers have successfully developed SLNs for the delivery of various ATDs including INZ. Bhandari and Kaur (2013) developed and characterized INZ-SLNs. Significantly enhanced relative bioavailability of INZ, 3 times higher $\mathrm{LD}_{50}$ and low incidence of hepatotoxicity were reported after oral administration of INZ-SLNs as compared to the free drug solution at the same dose [3]. Similarly, Khuller and Pandey (2005) developed inhalable INZ, rifampicin and pyrazinamide encapsulated solid lipid particle (SLP) by emulsion solvent diffusion technique and evaluated its chemotherapeutic potential. It showed improved mean residence time and drug bioavailability with reduced biochemical hepatotoxicity [17]. In another study, the same researchers developed INZ, rifampicin and pyrazinamide encapsulated SLNs meant for oral administration and reported with improved drug bioavailability and reduced biochemical hepatotoxicity [18]. Bhandari and Kaur (2013) developed INZ-SLNs with higher drug encapsulation efficiency by microemulsification method and characterized [19]. Thus, SLNs may be a suitable drug delivery system for INZ delivery that may not only reduce dose-dependent toxicities but also deliver the drug to the targeted site.

So far, no researchers have ever tried to develop sustained release, optimized INZ-SLNs with higher entrapment efficiency and size suitability for avoiding hepatic metabolism. So in the current study, attempts were made to develop and characterize INZ encapsulate SLNs with higher entrapment efficiency, sustained drug release ability with significant anti- 
tubercular activity and reduced toxicity for improving chemotherapeutic potential and better patient compliance.

\section{Experimental Materials}

Cadila Pharmaceuticals Ltd (Ahmedabad, India) provided INZ as a gift sample. Stearic acids (SA), Poloxamer-188 (POL), and Soya Lecithin (LEC) were obtained from Himedia (Mumbai). The 3-(4, 5-dimethylthiazol-2-yl)-2, 5-diphenyltetrazolium bromide (MTT) was obtained from Sigma-Aldrich. All the cell culture products including Dulbecco's modified eagle's medium (DMEM), fetal bovine serum (FBS), and antibiotic and antimycotic solution were purchased from GibcoTM (Thermo Fisher Scientific). Culture flask and well plates were from Nunc (Thermo Scientific). All the remaining chemicals consumed were of analytical quality.

\section{Culture}

The culture of M. tuberculosis H37Rv and INZ resistant strains were obtained from the National Institute for Research in Tuberculosis, Chennai, India. The cell line (J774A.1) was procured from the National Centre for Cell Sciences, Pune, India.

\section{Experimental design}

The $2^{3}$ factorial designs were applied to evaluate the effect of three factors along with their consequences for the physicochemical property of SLNs. For the optimization of SLNs, the ratios of LEC : SA (A), drug : SA (B) and POL (C) had opted as independent variables. Each one factor was set with its minimum and maximum levels as illustrated in Table 1. A total of eight SLNs formulations (F1-F8) were prepared in accordance with the factorial design. The particle size (PS), entrapment efficiency percentage (EE\%) and cumulative percentage drug release (CDR\%) were fixed as response parameters. Design Expert Software 10 (Stat-Ease Inc., USA) was employed to analyze the obtained data.

\section{Preparation of solid lipid nanoparticles (SLNs)}

SLNs were prepared by high pressure homogenization followed by ultrasonication technique. Based on the partitioning results, the combination of SA : LEC at $1: 0.5$ was selected as solid lipids for the formulation of INZ loaded SLNs (INZ-SLN). Briefly, molten lipid phase was obtained by heating lipids (SA and LEC) at $-10{ }^{\circ} \mathrm{C}$ exceeding the lipid melting temperature. An aqueous phase was formulated by dissolving the surfactants (POL) and drug in triple distilled water and heated up to the same temperature as that of lipid phase. Subsequently, the aqueous phase added to the lipid phase which was homogenized with a high pressure homogenizer (APV 2000, Rosista $\mathrm{GmbH}$ ) at 750 bar and 3 cycles. Soon after the homogenization, sonication (Ultrasonicator 300V/T, Biologics, Inc.) was done for $60 \mathrm{sec}$ at 40 pulses. Subsequently, the dispersions were allowed to solidify by keeping it in ice bath. The SLNs were collected after completion of solidification and were lyophilized in order to avoid a series of stability issues like aggregation, fusion and seepage of the encapsulated drug into the storage medium. A total of eight INZ-SLNs formulations (F1F8) were prepared and their detailed compositions are shown in Table 1.

Table $12^{3}$ Full-factorial design and characterization of prepared INZ-SLNs [mean $(n=3)$ ]

\begin{tabular}{cccccccccc}
\hline FC & LEC : SA (A) & D : SA (B) & POL (C) & PS & PDI & ZP & TDC\% & EE\% & CDR\% \\
\hline F1 & -1 & -1 & -1 & 142.7 & 0.195 & -29.0 & 94.16 & 64.12 & 64.16 \\
F2 & +1 & -1 & -1 & 130.6 & 0.219 & -32.7 & 91.32 & 69.16 & 69.32 \\
F3 & -1 & +1 & -1 & 184.2 & 0.235 & -29.6 & 93.75 & 67.37 & 73.34 \\
F4 & +1 & +1 & -1 & 167.1 & 0.229 & -32.4 & 93.85 & 73.17 & 79.14 \\
F5 & -1 & -1 & +1 & 135.6 & 0.247 & -30.4 & 90.45 & 61.52 & 71.92 \\
F6 & +1 & -1 & +1 & 120.2 & 0.199 & -33.9 & 91.78 & 66.34 & 78.24 \\
F7 & -1 & +1 & +1 & 165.5 & 0.286 & -30.8 & 90.26 & 63.45 & 76.15 \\
F8 & +1 & +1 & +1 & 145.6 & 0.243 & -34.8 & 92.84 & 68.05 & 82.36 \\
\hline
\end{tabular}

Note: $\mathrm{A},-1=0.25,+1=0.5 ; \mathrm{B},-1=0.1,+1=0.2 ; \mathrm{C},-1=1 \%,+1=2 \%$.

Abbreviations: $\mathrm{FC}=$ Formulation code; $\mathrm{LEC}=$ Soya lecithin; $\mathrm{SA}=$ Stearic acid; $\mathrm{D}=$ Drug (isoniazid); $\mathrm{POL}=\mathrm{Poloxamer} 188$; $\mathrm{PS}=\mathrm{Particle}$ size; $\mathrm{PDI}$

$=$ Polydispersity index; $\mathrm{ZP}=$ Zeta potential; $\mathrm{EE}=$ Entrapment efficiency; $\mathrm{TDC}=$ Total drug content; $\mathrm{CDR}=\mathrm{Cumulative}$ drug release. 


\section{Characterization of solid lipid nano- particles (SLNs)}

\section{Particle size (PS) and polydispersity index (PDI)}

90 s Plus Particle size analyzer (Brookhaven, New York, USA) with dynamic laser light scattering technique was employed in SLNs dispersions for assessing the mean PS and PDI. 100 times dilution of dispersions with deionized water was done and the measurements obtained by an angle of $90^{\circ}$.

\section{Zeta potential (ZP)}

Horiba, SZ-100 Scientific series, Japan was employed for the determination of the ZP of different formulations with the help of a laser light scattering technique. Double distilled water was used to dilute the formulations appropriately. Measurements were obtained with an angle of $90^{\circ}$.

\section{Total drug content (TDC) and entrapment efficiency (EE\%)}

The total quantity of drug per unit volume present in the INZ-SLNs was estimated by suitably disrupting $1 \mathrm{~mL}$ of the SLN dispersion in $5 \mathrm{~mL}$ chloroform: methanol (1:1) volumetrically. The amount of INZ was determined by ultraviolet (UV)-visible spectrophotometer at $262 \mathrm{~nm}$. Blank SLNs sample with similarly processing was taken as control. Each experiment was carried out in triplicate. The total drug content was determined by using the equation given below,

$$
\mathrm{TDC}=\frac{\text { Calculated amount of drug } / \mathrm{mL} \text { of SLN dispersion }}{\text { Total amount of drug added } / \mathrm{mL} \text { of SLN dispersion }} \times 100 .
$$

Remi cooling centrifuge (Mumbai) was used to analyse the clear supernants obtained by centrifuging the developed SLN dispersions for estimation of EE\%:

$\mathrm{EET}=\frac{\mathrm{TDC}-\mathrm{D}_{\mathrm{sf}}}{\mathrm{TDC}} \times 100$,

where $D_{s f}=$ quantity of drug in clear supernatant fluid.

\section{In vitro drug release study}

In vitro drug release study was performed by the dialysis bag diffusion method using dialysis membrane. The amount of SLNs containing $2.6 \mathrm{mg}$ of INZ was taken in the dialysis tube that immersed in a receptor compartment having $80 \mathrm{~mL}$ of dissolution media $(\mathrm{pH}$ 6.8 phosphate buffer maintained at $37 \pm 0.5^{\circ} \mathrm{C}$ ) and stirred continuously at $100 \mathrm{rpm}$ by a magnetic stirrer.
To prevent the loss of dissolution medium due to evaporation, the receptor compartment was wrapped with aluminum foil. Samples $(2 \mathrm{~mL})$ were withdrawn at specified time intervals $(1,2,3,4,6,8,10,12,18$, $24,36 \mathrm{~h}$ ) and in order to maintain the volume, an equal volume of fresh dissolution medium was added to the compartment. The amount of drug release was estimated using UV-visible spectrophotometer at 262 $\mathrm{nm}$ after appropriate diluting each withdrawn sample. The CDR\% from the different formulations was calculated. This experimentation was performed in triplicate.

\section{In vitro release kinetics}

The in vitro drug release kinetics of INZ-SLNs formulations was estimated. The drug release patterns were known by analyzing the obtained data. The release data were fitted to Zero order, First order, Higuchi, and Korsmeyer-Peppas kinetic models as follows (Eqs.(3)-(6)),

Zero order release model: $\mathrm{Q}=\mathrm{K}_{0} \mathrm{t}$,

First order release model: $\ln \mathrm{Q}_{\mathrm{t}}=\ln \mathrm{Q}_{0}-\mathrm{K}_{1} \mathrm{t}$,

Higuchi kinetic model: $\mathrm{Q}_{\mathrm{t}}=\mathrm{K}_{\mathrm{H}} \mathrm{t}^{1 / 2}$,

and

Korsmeyer and Peppas model: $\log \left(\mathrm{Q}_{\downarrow} / \mathrm{Q}_{\infty}\right)=\mathrm{nLog} \mathrm{t}+\log \mathrm{k}, \quad$ (6) where $\mathrm{Q}_{0}=$ quantity of drug present initially in SLNs, $\mathrm{Q}_{\mathrm{t}}=$ quantity of drug released at time $\mathrm{t}$ hour, $\mathrm{Q}_{\infty}=$ quantity of drug released at infinite time, and $\mathrm{Q}_{t} / \mathrm{Q}_{\infty}=$ fraction of drug released at time $\mathrm{t}, \mathrm{n}$ is the diffusion exponent, and $\mathrm{K}_{0}, \mathrm{~K}_{1}, \mathrm{~K}_{\mathrm{H}}, \mathrm{k}=$ release rate constants.

\section{Selection of an optimized formulation and stability study}

Optimized formulation was chosen on the basis of software analysis (Design Expert 10) in addition to overall desirability value [20]. This software tool offers the possibility to vary each variable parameter simultaneously and presents possible optimum. The optimum formulation was picked up based on the result obtained from it. In this study, we chose optimized formulation based on lower PS, higher EE\%, and higher CDR\% after $36 \mathrm{~h}$.

Stability study of the optimized formulation was carried out by keeping sealed vials containing SLNs in a stability chamber maintained at $5 \pm 3{ }^{\circ} \mathrm{C}$ and $25 \pm 2{ }^{\circ} \mathrm{C} / 60 \pm 5 \%$ relative humidity (RH). Sealed SLNs were evaluated for PS, EE\% over a period of six 
months with a sampling at 1, 2, 3, 6 months.

\section{Characterizations of optimized formulation Transmission electron microscopy (TEM)}

To study the morphology of SLNs dispersion, TEM (JEM-1200EX, JEOL, Japan) was employed. Diluted SLNs dispersion (single drop) was kept on a copper grid. Aqueous solution of phosphotungstate acid (2\% w/v) was used as contrast enhancer. The sample was dried up and scanned underneath the TEM.

\section{Fourier transform infrared (FT-IR) spectroscopy}

FT-IR (Agilent Resolution Pro) was employed to record and analyze the IR spectra of pure INZ, SA, and INZ-SLNs, in the range of $4000-600 \mathrm{~cm}^{-1}$ at room temperature.

\section{Differential scanning calorimeter (DSC) analysis}

The thermal study of INZ, SA and INZ-SLNs was carried out by using DSC (NETZSCH DSC 214 Polyma, Germany). About $10 \mathrm{mg}$ of samples were placed in aluminum crimp cells and heated from 30 to $400{ }^{\circ} \mathrm{C}$ with a scanning rate of $10^{\circ} \mathrm{C} / \mathrm{min}$ in a nitrogen ambiance.

The following equation was used for the estimation of percent crystallinity indexes (CI\%).

$$
\mathrm{CI} \%=\frac{\text { Enthalpy SLNs dispersion }(\mathrm{mJ} / \mathrm{mg})}{\begin{array}{c}
\text { Enthalpybulk materials }(\mathrm{mJ} / \mathrm{mg}) \times \\
\text { Concentration lipid phase }(\%)
\end{array}} \times 100 \text {. }
$$

\section{X-ray diffraction (XRD)}

The crystal behavior of SLNs was confirmed through XRD study using X-ray diffractometer (XPERT-PRO, PANalytical, Netherlands). It was performed by exposing the samples to $\mathrm{Cu} \mathrm{K}_{\alpha}$ radiation $\left(45 \mathrm{kV}, 40 \mathrm{~mA}\right.$ ) and scanning from $15^{\circ}$ to $90^{\circ}, 2 \theta$ at a step size of $0.010^{\circ}$. The instrument measures the scattering angle ' $\theta$ ' that helps in calculating the interlayer spacing ' $d$ ' value using Bragg's equation $n \chi=$ $2 \mathrm{~d} \sin \theta$, where ' $\mathrm{n}$ ' is the order of the interference and ' $\chi$ ' is the incident $\mathrm{X}$-ray beam wavelength. Comparison between the characteristic peak intensity of pure drug and the obtained XRD pattern was done.

\section{In vitro antitubercular activity of optimized formulation by Luciferase reporter phage (LRP) assay}

Preparation of cell suspension

Cell suspension equivalent to McFarland standard No. 2 was prepared by inoculating the log phase culture of standard mycobacterium tuberculosis strain H37Rv and INZ resistant strains from LöwensteinJensen (L-J) slope and G7H9 broth in Bijou bottles with 6-8 glass beads (diameter $=3 \mathrm{~mm}$ ) which was homogenized by vortexing for $15-20 \mathrm{sec}$. The cell suspensions containing H37Rv and INZ resistant strain were incubated at $37^{\circ} \mathrm{C}$ for $72 \mathrm{~h}$.

Luciferase reporter phage (LRP) assay

Antimycobacterial activities of the INZ-SLN and INZ aqueous solution were evaluated by adopting LRP assay as described [21], with slight modifications. Approximately, $350 \mu \mathrm{L}$ of G7H9 broth with $10 \%$ albumin-dextrose complex and $0.5 \%$ glycerol were taken in cryo vials, to which INZ-SLNs suspensions were added to achieve a final INZ concentration of $100 \mu \mathrm{g} / \mathrm{mL}$. Lastly, $100 \mu \mathrm{L}$ of MTb cell suspensions were added to all the vials. The same test procedure was followed for all isolates of MTb used. In the assay, DMSO (1\%) was used as solvent control. All the vials were incubated for $72 \mathrm{~h}$ at $37{ }^{\circ} \mathrm{C}$. After incubation, $50 \mu \mathrm{L}$ of high titre mycobacteriophage phAETRC202 and $40 \mu \mathrm{L}$ of $0.1 \mathrm{M} \mathrm{CaCl}_{2}$ solution were added into the INZ-SLNs and INZ solution containing vials. All the vials were incubated at $37^{\circ} \mathrm{C}$ for $4 \mathrm{~h}$. After incubation, $100 \mu \mathrm{L}$ of the sample from each vial was transferred to luminometer cuvette and an equal amount of D-Luciferin $(0.3 \mathrm{mM}$ in $0.05 \mathrm{M}$ sodium citrate buffer, $\mathrm{pH}$ 4.5) was added. The relative light unit (RLU) was measured in a luminometer (Monolight 2010, Ellsworth Rd, Arizona, USA).

RLU reduction $\%=\frac{\text { Control RLU }- \text { Test RLU }}{\text { Control RLU }} \times 100$.

Sample showing RLU reduction by $50 \%$ or more when compared to control was considered as having antimycobacterial activity.

\section{Cytotoxicity studies of optimized formulation}

MTT assay method

MTT assay with some modifications was followed to evaluate the viabilities of J774A.1 cells by detecting functioning mitochondria [22]. Live mitochondria convert MTT to insoluble purple formazan crystals, which could be measured spectrophotometrically at $590 \mathrm{~nm}$. In each well of a $96-$ well plate, $100 \mu \mathrm{L}$ of $1 \times 10^{6}$ cells $/ \mathrm{mL}$ was cultured and allowed to grow overnight at $37{ }^{\circ} \mathrm{C}$, in an environment containing $5 \%$ $\mathrm{CO}_{2}$ and $80 \%$ humidity incubator. The next day, the media $(100 \mu \mathrm{L})$ was substituted with fresh media and $100 \mu \mathrm{L}$ of cell culture media containing either INZ or 
INZ-SLNs. Then the plate was incubated for $24 \mathrm{~h}$. A sterilized stock MTT solution $(10 \mu \mathrm{L}$ of $5 \mathrm{mg} / \mathrm{mL}$ in Dulbecco's phosphate buffer saline) was added to each well holding $150 \mu \mathrm{L}$ of new media and incubated for $4 \mathrm{~h}$ at $37^{\circ} \mathrm{C}$. Later, the supernatant was confiscated carefully and formazan crystals were dissolved by adding $100 \mu \mathrm{L}$ of DMSO. The absorbance was recorded spectrophotometrically at $590 \mathrm{~nm}$ with an ELISA reader 113 (i MARK, Biorad, USA). The ratio of viable cells in the INZ-SLNs and free INZ-treated well were compared. The cell viability was estimated using the following formula,

Cell viability $(\%)=\frac{\text { Abs. sample }}{\text { Abs. control }} \times 100$.

The viability of untreated controls was normalized to $100 \%$ :

Cytotoxicity $(\%)=100 \%-$ Cell viability $(\%)$.

\section{Statistical analysis}

In the present study, all data were reported as the mean \pm standard deviation (SD). The significance of differences was evaluated using Student's t-test and one-way ANOVA at $\mathrm{P}<0.05$.

\section{Results and Discussion Particle size (PS), polydispersity index (PDI) and zeta potential (ZP)}

The PS, PDI and ZP values have been depicted in Table 1. Effects of the independent variables such as D (drug) : SA, LEC : SA and POL concentrations on particle size were studied (Fig. 1). The result showed that D : SA and POL\% were critical parameters in governing the particle size. Mean particle sizes of

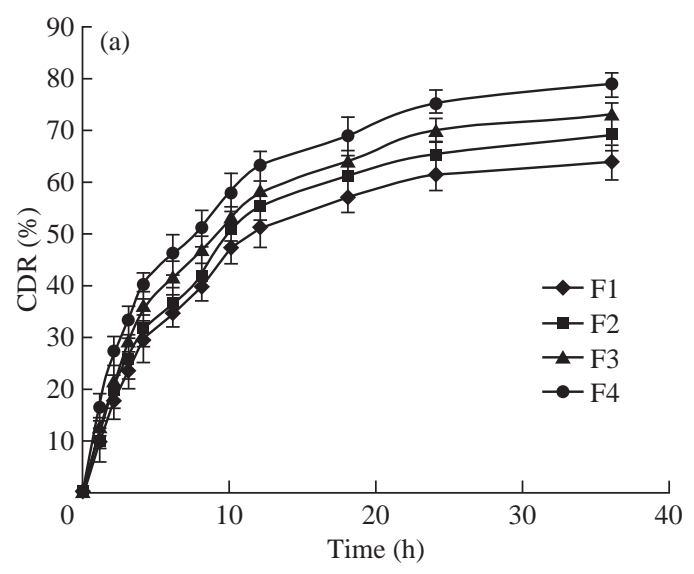

SLNs ranged between 120.2 and $184.2 \mathrm{~nm}$. The mean particle size was observed least with a high level of POL, LEC : SA ratio and low levels of D : SA ratio. However, the particle size was largest when D : SA ratio was at high level and both LEC : SA ratio and POL were at low level. Consequently, upon increasing the $\mathrm{POL} \%$ in the lipid matrix from $1 \%$ to $2 \%$, the mean particle size decreased. The response coefficients were determined for each dependent variable with a view to evaluate the effect of each response.

The particle size of SLNs was estimated using the following polynomial equation,

$\mathrm{PS}(\mathrm{nm})=$

148.94-8.06A+16.66B-7.21C-1.19AB-0.76AC-2.84 BC. (11)

PS is one of the most important characteristics of the nanoparticles, since the release pattern of the drug and its absorption depends on it. SLNs were prepared using high pressure homogenization followed by sonication method which helped in producing relatively smaller particles. Smaller particles (below $200 \mathrm{~nm}$ ) were preferred as they not only remained imperceptible to the reticulo-endothelial system (RES) [3], but also were suitable for macrophage uptake and for circulation over a protracted episode of time [23]. We were successful in getting all the particles of size below $200 \mathrm{~nm}$. With the increase in the lipid concentration, the particle sizes of INZ-SLNs increased as the higher tendency of lipid coalescence occurred at higher lipid concentration due to the internal and external phase density difference [24], which could be explained by Stoke's law. At high concentration, the viscosity of lipid increased which led to reducing the diffusion rate of the solute molecule into outer phase, resulting in increased particle size [25]. Moreover, with the increase in the LEC : SA ratio, the size of SLNs decreased. The particle size

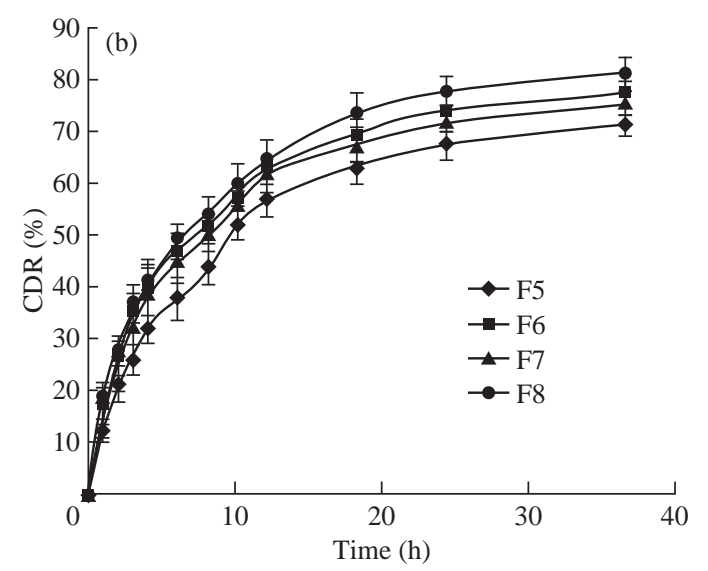

Fig. 1 In vitro release profiles of INZ SLNs up to 36 h: (a) F1-F4 and (b) F5-F8 in phosphate buffer, pH 6.8. 
decreased upon increasing the concentration of POL. This behavior can be explained by the surfactantinduced reduction in surface tension between aqueous phase and oil phase leading to the formation of smaller-size emulsion droplets. However, surfactant helps to stabilize the newly generated surfaces and prevent particle aggregation [26]. High recovery is a precondition for developing a manufacturing process of any formulation as it accounts for losses during the production process.

The PDIs for all the developed systems were between 0.195 and 0.286 , indicating a narrow particle size distribution. The PDIs of all the developed SLNs systems were $<0.3$, which indicated a narrow and homogeneous particle size distribution.

As shown in Table 1, the ZP values closely ranged between $-34.8 \mathrm{mV}$ and $-29 \mathrm{mV}$. The lowest value of $\mathrm{ZP}$ was reported for $\mathrm{F} 1$ and the highest for $\mathrm{F} 8$, and all the remaining formulations showed intermediate values. ZP value of the optimized formulation was found to be $-32.4 \mathrm{mV}$ (Fig. 2). The nanoparticles are thermodynamically unstable systems and a $\mathrm{ZP}$ value of equal to or more than $-30 \mathrm{mV}$ is desirable for the stability of colloidal drug carriers. ZP values of all prepared formulations were reported to be significantly negative $(\mathrm{p}<0.05)$, and thus predicted high particle stability and limited aggregation with prolonged storage. LEC and POL were the main components in the formulations that influenced the $\mathrm{ZP}$. The negative charge of ZP values may be attributed to LEC, as it is an anionic surfactant and develops electrostatic repulsion between particles resulting in reducing the

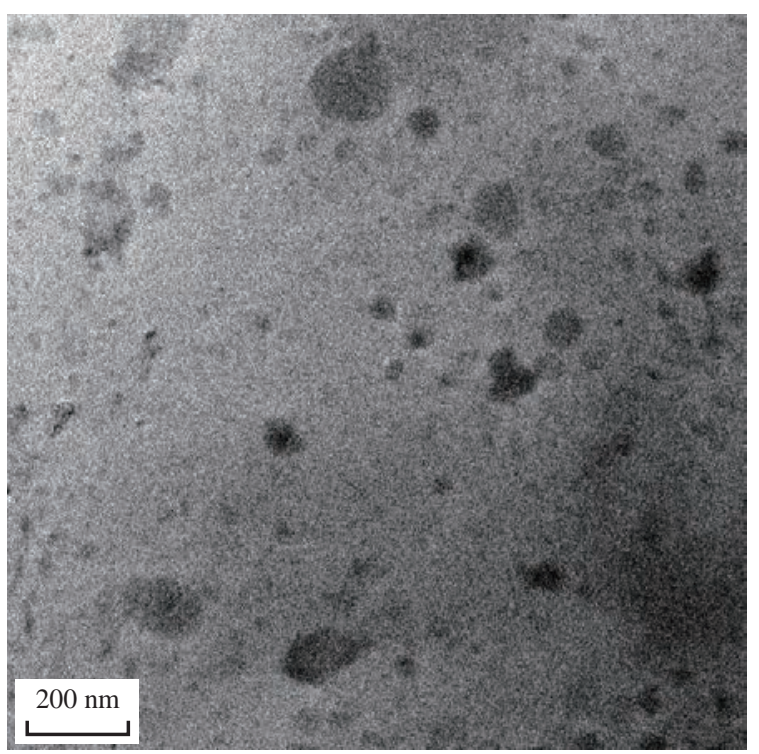

Fig. 2 Transmission electron microscopy of optimized INZSLNs. aggregation [27]. ZP of the formulations decreased with increase in the LEC : SA ratio. Moreover, on the increase in \% POL, the $\mathrm{ZP}$ value decreased as $\mathrm{POL}$ is a non-ionic surfactant.

\section{Entrapment efficiency (EE\%) and total drug content (TDC)}

A very important matter regarding the utilization of lipid nanoparticles as drug carriers is their aptitude for drug loading, and therefore the effects of independent variables such as POL\%, D : SA and LEC : SA ratios on drug entrapment efficiency were investigated (Fig. 1). The highest EE\% (73.17\%) was observed with high levels of D : SA, LEC : SA ratios and a low level of POL concentration, whereas the lowest EE\% (61.52\%) was obtained with a higher POL concentration with lower LEC : SA and D : SA ratios. The response coefficients were determined for each dependent variable in order to evaluate the effect of each response. EE\% of SLNs was estimated using the following polynomial equation,

$\mathrm{EE} \%=$

$66.77+2.66 \mathrm{~A}+1.49 \mathrm{~B}-1.68 \mathrm{C}+0.19 \mathrm{AB}-0.052 \mathrm{AC}-0.33 \mathrm{BC}$.

All independent variables showed significant and indirect correlations with entrapment efficiency. With the increase in the level of lipid, the EE\% increased as the higher concentration of lipid led to the formation of bigger-size SLNs particles which provided some additional space to entrap the drug molecules. Higher $\mathrm{EE} \%$ was obtained at the higher level of LEC/SA, owing to the higher surfactant behavior of LEC, and the hydrophilic interaction between drug and LEC leading to higher incorporation of hydrophilic drug into the lipid. A higher level of POL resulted in lower EE\% value, which may be owing to the higher influence of surfactant behavior that enhanced the higher aqueous solubility of drug [28].

All the SLN formulations exhibited a TDC of $>90 \%$ $\mathrm{w} / \mathrm{v}$. The above $90 \%$ TDC values confirmed the aptness of the developed process and its pilot scale feasibility.

\section{In vitro release studies and release kinetics}

In an attempt to develop an extended release system, it is imperative to be aware of the release mechanism and kinetics. The effect of independent variables on CDR\% in $36 \mathrm{~h}$ was studied (Fig. 1). The highest $\mathrm{CDR} \%(82.36 \%)$ was observed with a higher concentration of POL and higher D : SA and LEC: SA 
ratios, and vice versa $(64.16 \%)$. The release profiles were identified as biphasic with initial faster release phase till $10 \mathrm{~h}$, followed by a slower release phase (Fig. 1). The CDR\% of SLNs was estimated using the following polynomial equation,

$\mathrm{CDR} \%=$

$+74.33+2.94 \mathrm{~A}+3.42 \mathrm{~B}+2.84 \mathrm{C}+0.066 \mathrm{AB}+0.20 \mathrm{AC}-1.33 \mathrm{BC}$.

On increase in the ratios of both D : SA and LEC : SA, the CDR\% increased, but in the case of D : SA ratio, it was comparatively higher. Moreover, with increase in the concentration of POL (1-2\%), the CDR\% increased. The in vitro release of INZ-SLNs exhibited bi-phasic release characteristics with an initial burst effect followed by controlled release. The burst effect may be attributed to the unbound drug and the drug molecules that were adsorbed on the SLNs surface. Controlled release pattern depends on the rate of release of the entrapped INZ that further depends on the degree of solubility and rate of diffusion of INZ from the lipid matrix. Diffusion of INZ from lipid matrix also depends on the partitioning of INZ between the lipid phase and the aqueous dissolution media. The same pattern of release was also reported for gemcitabine [29].

The in vitro release data of INZ from SLNs were fitted into Zero order, First order, Higuchi, KorsmeyerPeppas models to study the drug release behavior. The release kinetics data are shown in Table 2. On comparing the $\mathrm{R}^{2}$ values of the SLNs, it was observed that it followed Higuchi and Korsmeyer-Peppas equations. However, it was best fitted to KorsmeyerPeppas model. The release exponent ' $n$ ' was lower than 0.45, which implied Fickan diffusion mechanism of release of INZ from SLN. The response coefficient was determined for each dependent variable in order to evaluate the effect of each response. As the release pattern best fitted to Korsmeyer-Peppas model and according to the ' $n$ ' values of KorsmeyerPeppas model, almost all the formulations were shown to follow Fickian diffusion. For the optimized formulation $51 \%$ of the drug was released at the end of $8 \mathrm{~h}$, and after about $36 \mathrm{~h}$ drug release reached a steady state (79\%). This validated that the prepared INZSLN could sustain the drug release into the systemic circulation and hypothetically be able to lower the dosing frequency.

\section{Optimized formulation and stability study}

Though there were three parameters for selection of the optimized formulation of prepared INZ-SLNs, the PS result indicated that all the formulations were below $200 \mathrm{~nm}$, and thus were suitable for bypassing reticuloendothelial systems (RES). Hence in our present study, PS parameter was not considered for selection of optimized formulations. However, the remaining two parameters such as higher EE\% and higher CDR\% were considered for the selection of optimized formulations. It was found that $\mathrm{F} 4$ had the highest EE\% and a higher CDR\%. The EE\% and CDR\% of F6 were lower than F4. Similarly, though F8 had the highest $\mathrm{CDR} \%$, its EE\% was lower than F4. Moreover, among all the formulations, F4 showed the highest desirability value (0.93). Based on the obtained results and the desirability value, F4 was selected as the optimized formulation and considered batch fulfilling all the constraints favorable for the preparation of INZSLNs.

Table 2 In vitro release kinetics of INZ- SLNs formulations up to $36 \mathrm{~h}$

\begin{tabular}{|c|c|c|c|c|c|c|c|c|c|}
\hline \multirow{2}{*}{ FC } & \multicolumn{2}{|c|}{ Zero order } & \multicolumn{2}{|c|}{ First order } & \multicolumn{2}{|c|}{ Higuchi model } & \multicolumn{3}{|c|}{ Korsemayer peppas model } \\
\hline & $\mathrm{K}_{0}$ & $\mathrm{R}^{2}$ & $\mathrm{~K}_{1}$ & $\mathrm{R}^{2}$ & $\mathrm{~K}_{\mathrm{H}}$ & $\mathrm{R}^{2}$ & $\mathrm{Kp}$ & $\mathrm{R}^{2}$ & $\mathrm{n}$ \\
\hline $\mathrm{F} 1$ & 1.65 & 0.754 & 0.027 & 0.848 & 11.73 & 0.944 & 13.50 & 0.961 & 0.392 \\
\hline $\mathrm{F} 2$ & 1.79 & 0.755 & 0.032 & 0.872 & 12.73 & 0.943 & 12.88 & 0.933 & 0.427 \\
\hline F3 & 1.83 & 0.743 & 0.034 & 0.878 & 13.10 & 0.942 & 16.44 & 0.950 & 0.410 \\
\hline F4 & 1.92 & 0.732 & 0.041 & 0.901 & 13.75 & 0.940 & 20.04 & 0.968 & 0.397 \\
\hline F5 & 1.84 & 0.761 & 0.034 & 0.874 & 13.06 & 0.948 & 15.10 & 0.964 & 0.429 \\
\hline F6 & 1.87 & 0.709 & 0.041 & 0.837 & 13.52 & 0.926 & 20.84 & 0.946 & 0.396 \\
\hline F7 & 1.85 & 0.718 & 0.039 & 0.868 & 13.34 & 0.930 & 18.92 & 0.948 & 0.411 \\
\hline F8 & 1.98 & 0.726 & 0.046 & 0.912 & 14.26 & 0.937 & 21.92 & 0.965 & 0.361 \\
\hline
\end{tabular}

Note: $\mathrm{K}=$ Rate constant $\left(\mathrm{K}_{0}\right.$, Zero order; $\mathrm{K}_{1}$, First order, $\mathrm{K}_{\mathrm{H}}$, Higuchi; $\mathrm{K}_{\mathrm{p}}$, Korsemayer-Peppas). 
Stability study results showed that the mean diameters of INZ-SLNs increased from 167.1 to 183.6 $\mathrm{nm}$ at $5 \pm 3{ }^{\circ} \mathrm{C}$, whereas it increased up to $210.3 \mathrm{~nm}$ at $25 \pm 2{ }^{\circ} \mathrm{C}, 60 \pm 5 \% \mathrm{RH}$ after completion of a 6 -month study period. The EE\% decreased by $5.55 \%$ at $5 \pm 3{ }^{\circ} \mathrm{C}$, whereas it was reduced by $9.79 \%$ at 25 $\pm 2{ }^{\circ} \mathrm{C}, 60 \pm 5 \% \mathrm{RH}$ after a 6-month storage period. Hence, the stability study indicated that at both temperatures, INZ-SLNs showed good stability, though there was a decrease in EE\% and an increase in PS. A comparatively higher reduction of EE\% of lyophilized INZ-SLNs appeared at $25 \pm 2{ }^{\circ} \mathrm{C}, 60 \pm 5 \% \mathrm{RH}$ as compared to $5 \pm 3^{\circ} \mathrm{C}$.

\section{Transmission electron microscopy (TEM)}

The SLNs were found spherical in shape with nanometric size, which was revealed from TEM images, as shown in Fig. 2. The particle size of SLNs measured by both TEM and dynamic light scattering technique was shown in good agreement.

\section{Fourier transmission infrared spectroscopy (FTIR)}

FTIR spectra were collected to confirm the presence of INZ in INZ-SLNs. Comparisons of FTIR spectra for free INZ, SA and INZ-SLNs showed the presence of INZ in SLNs (Fig. 3). The characteristic FTIR bands of INZ at $3105 \mathrm{~cm}^{-1}$ was assigned to $\mathrm{N}-\mathrm{H}$ stretching vibrations of the hydrazide group. Some other characteristic peaks were found at 1668 and $1564 \mathrm{~cm}^{-1}$, which was assigned to the hydrazide $\mathrm{C}=\mathrm{O}$ and the pyridine ring stretches, respectively. This confirmed that INZ was compatible with SA and the INZ remained stable during SLNs formulation. Presence of characteristics peaks of INZ in INZ-SLNs confirmed that INZ was compatible with SA and the INZ remained stable during SLNs formulation.

\section{Differential scanning calorimeter (DSC) studies}

The DSC thermograms of pure INZ, SA and INZSLNs are shown in Fig. 4. The pure INZ showed a single sharp endothermic peak at about $170.5{ }^{\circ} \mathrm{C}(\Delta \mathrm{H}$ $=260.1 \mathrm{~J} / \mathrm{g})$, whereas SA showed an endothermic peak at $56.3{ }^{\circ} \mathrm{C}(\Delta \mathrm{H}=194.4 \mathrm{~J} / \mathrm{g})$. A significant decrease in enthalpy of the peak was observed for INZ-SLNs (158.5 $\mathrm{J} / \mathrm{g}$ ), but the INZ peak was not visible in the SLNs thermogram. This specified that the INZ was not in crystalline form and was molecularly homogeneously dispersed in the nanoparticle matrix. The CI\% of the optimized formulation was calculated and found to be $54.35 \%$ against the reference value of $100 \%$ for SA. It was confirmed that the crystallinity of optimized SLNs formulation was depressed by the addition of the

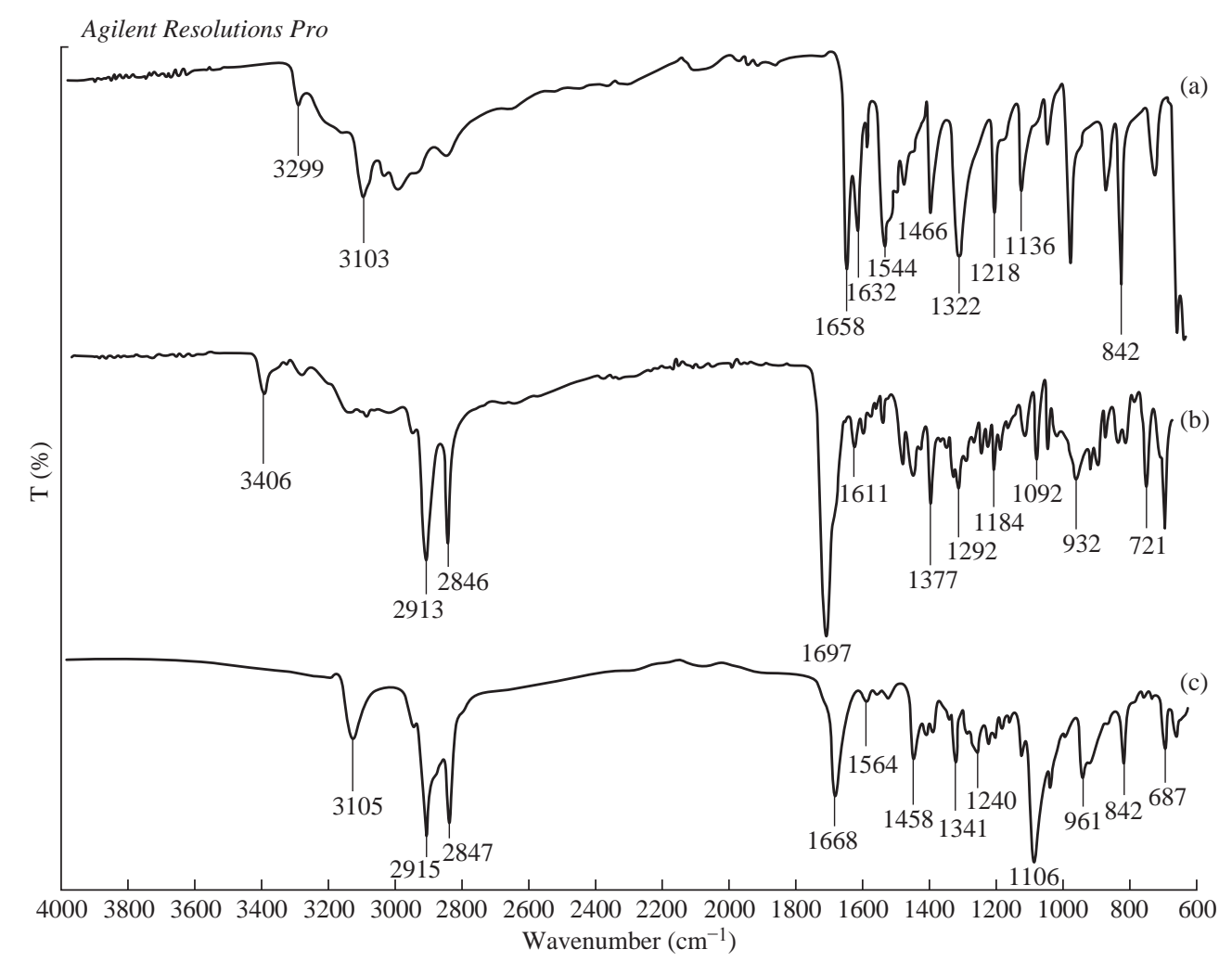

Fig. 3 Fourier transmission infrared spectrum of (a) free INZ, (b) stearic acid (SA), and (c) INZ-SLNs (F4). 


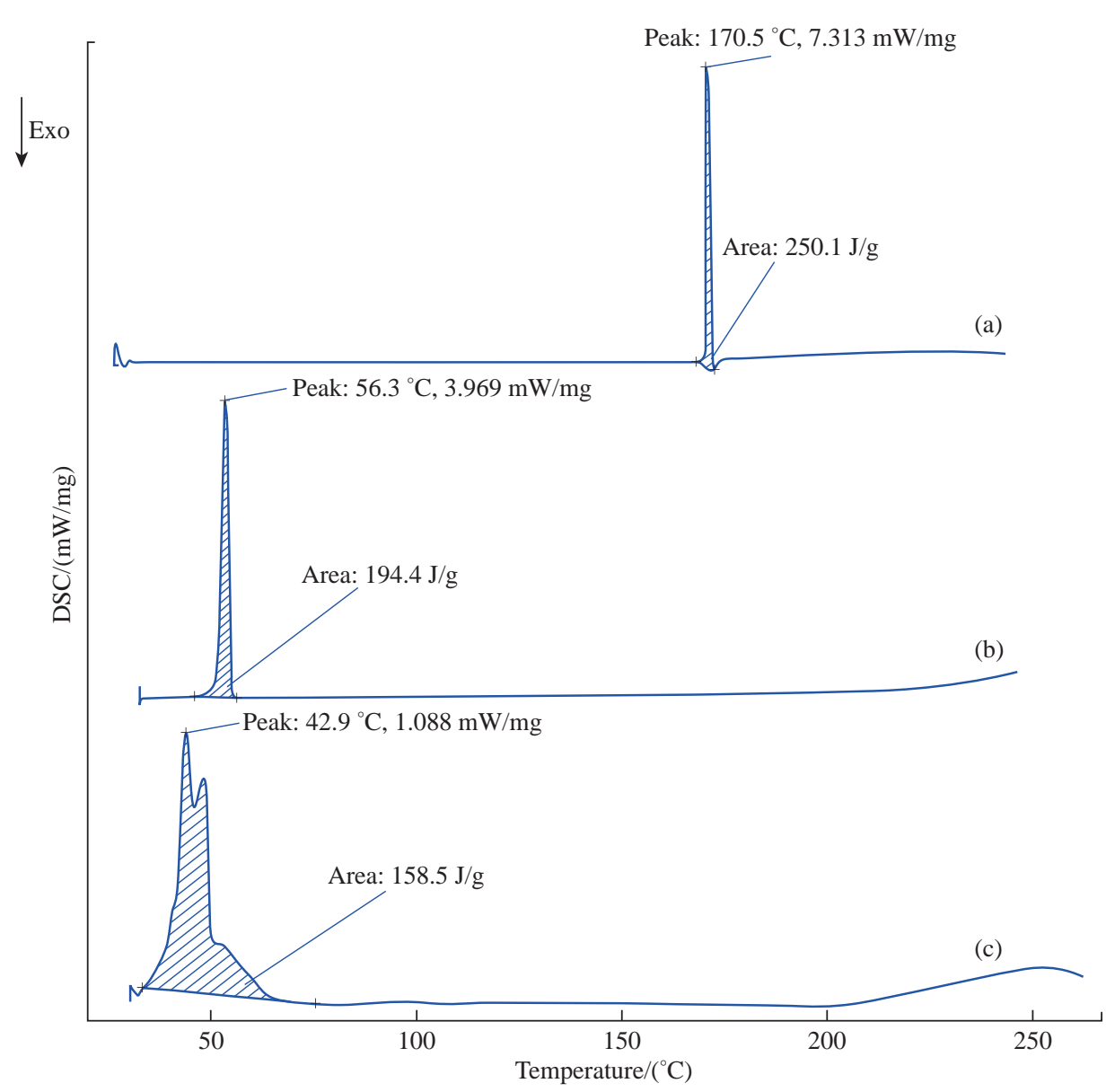

Fig. 4 Differential scanning calorimeter thermo grams of (a) isoniazid (INZ), (b) stearic acid (SA) and (c) INZ-SLNs (F4).

LEC and POL that promoted disordered arrangement resulting in reduced recrystallization tendency of solid lipid. The depression in melting point (MP) may be owing to its small particle size, high specific surface area and the existence of surfactant. The solubility of drug in lipid and the presence of lipid imperfections (indicating spaces for drug incorporation into lipidic crystal lattice) are two important factors for monitoring drug loading.

In the current study, the results were examined for a drop off in enthalpy change that correlated with the generation of imperfections (loss of crystallinity) or places to better accommodate the drug molecule within the lipid crystals. During SLNs preparation, in order to retain the drug molecules within the solid lipid corpse, a reduction in crystallinity must be maintained. Distortion of crystallization and lowering of melting enthalpy were noticed during the production of SLNs, owing to the transformation of bulk lipid into the nanoparticulate form that led to high specific surface area (lower PS and lower MP) and interaction of solid lipid with surfactant

\section{X-Ray diffraction (XRD)}

XRD patterns of INZ, SA and INZ-SLN are shown in Fig. 5. XRD pattern of INZ exhibited sharp peaks at $2 \theta$ scattered angles of $15.239,15.666,19.717,27.316$ and 28.937, indicating a crystalline nature. However, amorphous halo was obtained in all the SLNs with a degree of amorphicity decreasing as evidenced by the presence of peaks at $2 \theta$ scattered angles of 21.4 (insignificant). The diffraction spectrum of pure INZ showed that the drug was crystalline in nature with many distinct peaks. From the XRD study, SLNs showed an absence of all the major characteristic peaks of INZ, indicating its large amorphousness upon incorporation into lipid matrix. An amorphous or metastable form will dissolve at the fastest rate in lipid, because of its higher internal energy which enhances thermodynamic properties compared to crystalline materials. The extent of drug encapsulation and rate of drug release depend on the polymorphic behavior and crystallinity of SLNs which could be modified by the presence of surfactants, method of fabrication and size. 


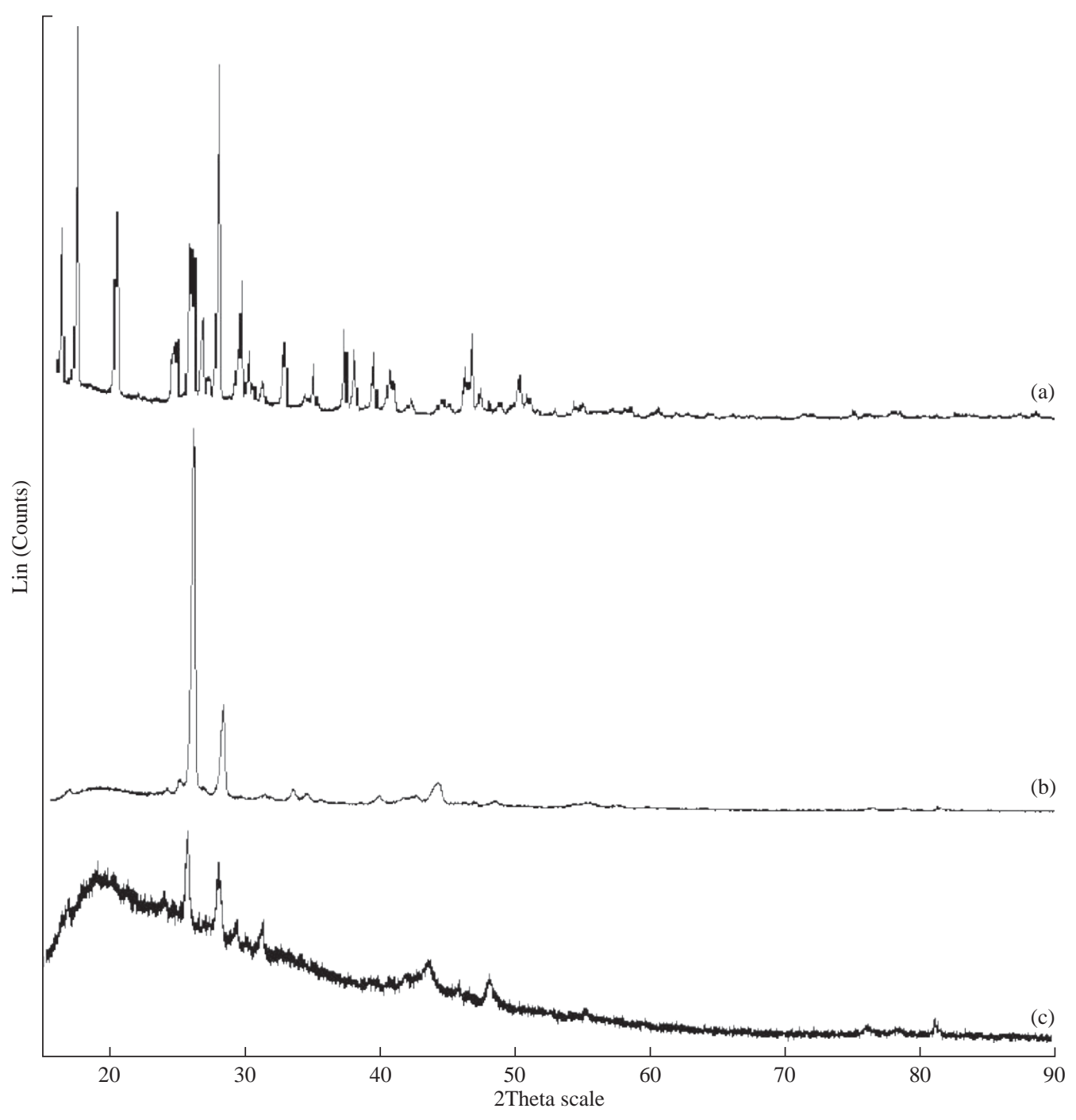

Fig. 5 X-ray diffraction spectrums of isoniazid (INZ), stearic acid (SA) and INZ-SLNs (F4).

\section{Luciferase reporter phage (LRP) assay}

LRP assay is a clinically established method for scrutinizing anti-tubercular efficacy of drugs in $H 37 \mathrm{R}_{\mathrm{V}}$ as well as isoniazid-resistant strain. A reduction of $50 \%$ RLU in the presence of test sample when compared with standard free control is considered as a significant anti-mycobacterial activity. The percentage reduction in RLU in the case of INZ at $0.1 \mu \mathrm{g} / \mathrm{mL}$ was found to be $99.898 \%$, whereas in the case of INZ-SLN (at INZ $0.1 \mu \mathrm{g} / \mathrm{mL}$ concentration) was $99.75 \%$. Moreover, in the case of INZ-resistant strain, the percentage reductions in RLU of INZ and INZ-SLNs at $0.1 \mu \mathrm{g} /$ mL concentration were 90.52 and $90.27 \%$ respectively. There was no significant increase in RLU on further increase in the drug concentration for both INZ and INZ-SLNs. The inhibitory concentration of the INZ loaded SLN was effective against MTb as well as its INZ resistant strain, and it was proved that lipid encapsulated INZ had retained nearly the same in vitro anti-TB activity as that of INZ.

\section{MTT assay}

The viabilities of J774A.1 cell line were determined after being challenged upon $24 \mathrm{~h}$ exposure with a different INZ concentration (0-20 mM) of both INZ and INZ-SLNs, and the impacts are shown in Fig. 6. At $20 \mathrm{mM}$ concentration of INZ, the J774A.1 cell viability after being exposed to free INZ was lower than $80 \%$, while the cell viability was higher than $90 \%$ in the case of INZ SLNs. Cell viability after being exposed to INZ-SLNs was higher than that for free INZ. These results indicated that INZ-SLNs were fairly biocompatible with the J774A.1 cell line and could be beneficial to reduce drug toxicity to normal cells. 


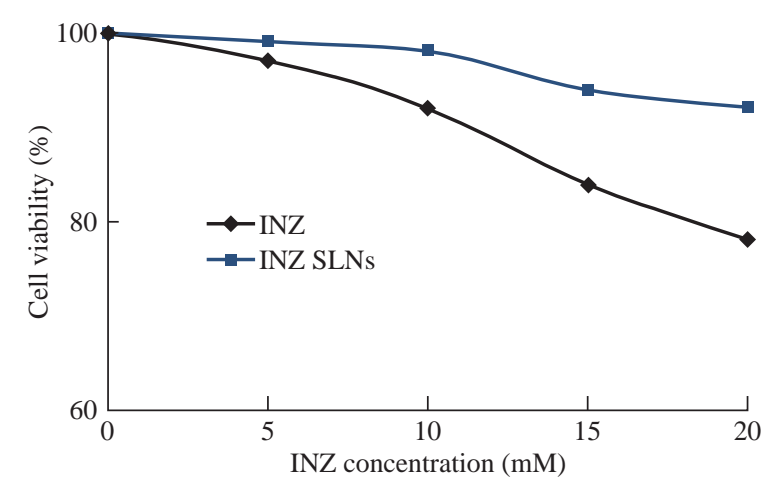

Fig. 6 Change of J774. A1 cell viability after $24 \mathrm{~h}$ treatment with free isoniazid (INZ) and lyophilized INZ- SLNs at equivalent INZ concentrations of 0-20 mM.

\section{Conclusions}

We have successfully developed INZ-SLNs and found small particle size with the spherical shape and optimum zeta potential. The developed SLNs were rationally designed and tailored to be favorable for bypassing RES and long-term controlled release of drugs. The in vitro release kinetics revealed that the release of INZ from SLNs followed Fickian's mechanism. The initial burst release of INZ might help in achieving minimum therapeutic concentration and the secondary controlled release characteristic helped in maintaining the therapeutic concentration. Results of LRP assay showed INZ-SLNs with significant in vitro anti tubercular activity. Cytotoxicity study (MTT assay) revealed that the INZ-SLNs were comparatively lesser toxic to normal cells as compared to free INZ. Thus, SLNs could be employed for delivery of INZ to achieve sustained drug release in systemic circulation and to lower dosing frequency with promising antitubercular activity for effective TB therapy.

\section{Conflicts of interest}

The authors confirm that this article content has no conflicts of interest.

\section{Acknowledgements}

We are thankful to Cadila Pharmaceuticals, Ahmadabad, India for providing us gift sample of Isoniazid.

\section{References}

[1] N. Fogel, Tuberculosis: A disease without boundaries.
Tuberculosis, 2015, 95(5): 527-531.

[2] M. Sedlak, D.S. Bhosale, L. Benes, et al., Synthesis and characterization of a $\mathrm{pH}$-sensitive conjugate of isoniazid with $\mathrm{Fe}_{3} \mathrm{O}_{4} @ \mathrm{SiO}_{2}$ magnetic nanoparticles. Bioorganic \& Medicinal Chemistry Letter, 2013, 23: 4692-4695.

[3] R. Bhandari, I.P. Kaur, Pharmacokinetics, tissue distribution and relative bioavailability of isoniazidsolid lipid nanoparticles. International Journal of Pharmaceutics, 2013, 441(1-2): 202-212.

[4] T. Scior, S.J. Garces-Eisele, Isoniazid is not a lead compound for its pyridyl ring derivatives, isonicotinoyl amides, hydrazides, and hydrazones: a critical review. Currrent Medicinal Chemistry, 2006, 13(18): 2205-2219.

[5] Z. Ahmad, R. Pandey, S. Sharma, et al., Alginate nanoparticles as antituberculosis drug carriers: Formulation development, pharmacokinetics and therapeutic potential. The Indian Journal of Chest Disease \& Allied Sciences, 2006. 48: 171-176.

[6] S. Azarmi, W.H. Roa, and R. Lobenberg, Targeted delivery of nanoparticles for the treatment of lung diseases. Advanced Drug Delivery Reviews, 2008, 60(8): 863-875.

[7] R. Pandey, S. Sharma, and G.K. Khuller, Oral poly(lactide-co-glycolide) nanoparticle based antituberculosis drug delivery: Toxicological and chemotherapeutic implications. Indian Journal of Experimental Biology, 2006, 44 (06): 459-467.

[8] N. Voigt, P. Henrich-Noack, S. Kockentiedt, et al., Toxicity of polymeric nanoparticles in vivo and in vitro. Journal of Nanoparticular Research, 2014, 16(6): 1-22.

[9] H.J. Yang, W.G. Cho, and S.N. Park, Stability of oilin-water nano emulsions prepared using the phase inversion composition mthod. Journal of Industrial and Engineering Chemistry, 2009, 15(3): 331-335.

[10] H. Singh, R. Bhandari, and I.P. Kaur, Encapsulation of Rifampicin in a solid lipid nanoparticulate system to limit its degradation and interaction with Isoniazid at acidic pH. International Journal of Pharmaceutics, 2013, 446: 106-111.

[11] D. Liu, C. Liu, and W. Zou, Enhanced gastrointestinal absorption of N3-O-toluyl-fluorouracil by cationic solid lipid nanoparticles. Journal of Nano Research, 2009, 1: $1-10$.

[12] S. Wang, R. Chen, Y. Wang, et al., Oridonin loaded solid lipid nanoparticles enhanced antitumor activity in MCF-7 cells. Journal of Nanomaterials, 2014, 2014: 1-11.

[13] M.H.A. Rashid, P.V.P.D. Bharadwaj, and V. Mandal, Preparation and characterization of PLGA loaded nanoparticles obtained from D. melanoxylon Roxb. leaves for their antiproliferative and antidiabetic activity. International Journal of Green Pharmacy, 2017, 11(3): S438-S447.

[14] J. Wang, H. Wang, and R. Zhu, Anti inflammatory activity of cuccumin loaded solid lipid nanoparticlesin IL-1 $\beta$ transgenic mice subjected to the lipopolysaccharide induced sepsis. Biomaterials, 2015, 53: 475-483.

[15] J.A. Loureiro, S. Andrade, A. Duarte, et al., Resveratrol and grape extract-loaded solid lipid nanoparticles for the treatment of Alzheimer's disease. Molecules, 2017, 22: 277.

[16] H. Harde, M. Das, and S. Jain, Solid lipid nanoparticles: An oral bioavailability enhancer vehicle. Expert Opinion on Drug Delivery, 2011, 8(11): 1407-1424.

[17] R. Pandey, G.K. Khuller, Solid lipid particle-based inhalable sustained drug delivery system against experimental tuberculosis. Tuberculosis, 2005, 85: 227 234

[18] R. Pandey, S. Sharma, and G.K. Khuller, Oral solid lipid nanoparticle-based antitubercular chemotherapy. Tuberculosis, 2005, 85: 415-420. 
[19] R. Bhandari, I.P. Kaur, A method to Prepare solid lipid nanoparticles with improved entrapment efficiency of hydrophilic drugs. Current Nanoscience, 2013, 9(2): 211220.

[20] Z. Wei, J. Hao, S. Yuan, et al., Paclitaxel-loaded Pluronic P123/F127 mixed polymeric micelles: formulation, optimization and in vitro characterization. International Journal of Pharmaceutics, 2009, 376(1-2): 176-185

[21] P.M. Sivakumar, P.S. Seenivasan, V. Kumar, et al., Synthesis, antimycobacterial activity evaluation and QSAR studies of chalcone derivatives. Bioorganic \& Medicinal Cheistry Letters, 2007, 17(6): 1695-1700.

[22] M. Singh, P. Sasi, G. Rai, et al., Studies on toxicity of antitubercular drugs namely isoniazid, rifampicin, and pyrazinamide in an in vitro model of HepG2 cell line. Medicinal Chemistry Research, 2011, 20(9): 1611-1615.

[23] S. Mustafa, V. Kusuma Devi, and R.S. Pai, Kanamycin sulphate loaded PLGA-vitamin-E-TPGS long circulating nanoparticles using combined coating of PEG and watersoluble chitosan. Journal of Drug Delivery, 2017, 2017: $1-10$.

[24] N.N. Palei, M.K. Das, Formulation, optimization and evaluation of solid lipid nanoparticles of lornoxicam. Latin American Journal of Pharmacy, 2013, 32(10): 1528-1537.

[25] J. Emami, H. Mohiti, H. Hamishehkhar, et al., Formulation and optimization of solid lipid nanoparticle formulation for pulmonary delivery of budesonide using Taguchi and BoxBehnken design. Research in Pharmaceutical Sciences, 2015, 10(1): 17-33.

[26] R.P. Thatipamula, C.R. Palem, R. Gannu, et al., Formulation and in vitro characterization of domperidon loaded solid lipid nanoparticles and nanostructure lipid carriers. DARU, 2011, 19(1): 23-32.

[27] S.M. de Carvalho, C.M. Noronha, C.L. Floriani, et al., Optimization of $\alpha$-tocopherol loaded solid lipid nanoparticles bycentral composite design. Industrial Crops and Products, 2013, 49: 278-285.

[28] M. Bhalekar, P. Upadhaya, and A. Madgulkar, Formulation and characterization of solid lipid nanoparticles for an anti-retroviral drug darunavir. Applied Nanoscience, 2017, 7: 47-57.

[29] P.T. Nandini, R.C. Doijad, H.N. Shivakumar, et al., Formulation and evaluation of gemcitabine-loaded solid lipid nanoparticles. Drug Delivery, 2015, 22 (5): 647-651.

Copyright $\subseteq$ Bibhash Chandra Mohanta, Subas Chandra Dinda, Gitanjali Mishra, Narahari Narayan Palei, and Vijayan Nynar Azger Dusthackeer. This is an open-access article distributed under the terms of the Creative Commons Attribution License, which permits unrestricted use, distribution, and reproduction in any medium, provided the original author and source are credited. 